04

\title{
Анизотропия анионной проводимости в монокристаллах суперионного проводника $\mathrm{CeF}_{3}$
}

\author{
(C) Н.И. Сорокин, В.В. Гребенев, Д.Н. Каримов \\ Институт кристаллографии им. А.В. Шубникова ФНИЦ „Кристаллография и фотоника“ РАН, \\ Москва, Россия \\ E-mail: nsorokin1@yandex.ru
}

Поступила в Редакцию 14 марта 2021 г.

В окончательной редакции 14 марта 2021 г.

Принята к публикации 22 марта 2021 г.

\begin{abstract}
Впервые изучена анизотропия анионной проводимости в кристаллах суперионного проводника $\mathrm{CeF}_{3}$ со структурой тисонита (пр. гр. $P \overline{3} c 1)$. Температурные $(300-600 \mathrm{~K})$ измерения проводимости проведены вдоль кристаллографических осей $a$ и $c$ тригональной элементарной ячейки кристалла. Максимальная величина электропроводности наблюдается вдоль оси $c$. Кристаллы суперионика $\mathrm{CeF}_{3}$ обладают слабой анизотропией электропроводности, $\sigma_{\| c} / \sigma_{\| a}=2.4, \sigma_{\| c}=5.6 \cdot 10^{-4} \mathrm{~S} / \mathrm{cm}$ при $500 \mathrm{~K}$. Обсуждается эффект анизотропии анионной проводимости в индивидуальных фторидах со структурой тисонита в связи с особенностями их атомного строения.
\end{abstract}

Ключевые слова: электропроводность, суперионные проводники, анизотропия, фториды редкоземельных элементов, фторид церия, структура тисонита.

DOI: 10.21883/FTT.2021.09.51313.054

\section{1. Введение}

Эффект анизотропии анионной проводимости во фторидах редкоземельных элементов (РЗЭ) со структурой тисонита (тип $\mathrm{LaF}_{3}$, пр.гр. $P \overline{3} c 1$ ), представляет большой интерес для изучения механизма ионного переноса во фторидных суперионных проводниках. Экспериментальные данные по измеренной в различных кристаллографических направлениях электропроводности тисонитовых суперионных кристаллов позволяют выявить структурные пути ионного транспорта и изучить взаимосвязь ионной проводимости и кристаллического строения.

Трифторид церия относится к группе трифторидов P3Э $R \mathrm{~F}_{3} \quad(R=\mathrm{La}, \mathrm{Ce}, \mathrm{Pr}$ и $\mathrm{Nd})$, которые обладают структурой тисонита и не претерпевают полиморфных превращений вплоть до плавления. Кристаллы $\mathrm{CeF}_{3}$ и твердые растворы на его основе рассматриваются как перспективные функциональные материалы для ионики (суперионные проводники, твердые электролиты) [1-3], фотоники (лазерные среды) и физики высоких энергий (сцинтилляторы) [4,5]. Крупногабаритные кристаллы трифторидов РЗЭ оптического качества могут быть выращены из расплава методами направленной кристаллизации, однако их получение и исследование свойств осложняется высокой реакционной способностью фторидов и их склонностью к пирогидролизу.

Анизотропия физических свойств кристаллов $R \mathrm{~F}_{3}$ исследована незначительно. К настоящему времени поведено исследование ионной проводимости только в кристаллах $\mathrm{LaF}_{3}$ [6-9] и $\mathrm{CeF}_{3}$ [8], ориентированных вдоль и перпендикулярно кристаллографической оси $c$.
Анизотропия механических свойств кристаллов $\mathrm{CeF}_{3}$ вдоль осей $a$ и $c$ изучена в [10], а анизотропия его оптических свойств исследовалась в [11].

Целью работы является исследование температурных зависимостей ионной проводимости тригональных кристаллов суперионного проводника $\mathrm{CeF}_{3}$ по разным кристаллографическим направлениям (оси $a$ и $c$ ) и рассмотрение эффекта анизотропии ионного переноса в тисонитовых трифторидах РЗЭ в связи с особенностями их кристаллического строения.

\section{2. Эксперимент}

Рост кристаллов $\mathrm{CeF}_{3}$ проводился из расплава методом вертикальной направленной кристаллизации во фторирующей атмосфере $\mathrm{CF}_{4}$ в установке с резистивным нагревом. В качестве исходной шихты использовался порошок $\mathrm{CeF}_{3}$ (99.99\%, ЛАНХИТ). Градиент температуры в ростовой зоне равен $100 \mathrm{~K} / \mathrm{cm}$, скорость вывода тигля из ростовой зоны составляла $\sim 5 \mathrm{~mm} / \mathrm{h}$. Охлаждение кристаллов проводили со скоростью $\sim 50 \mathrm{~K} / \mathrm{h}$. Получены прозрачные кристаллические були диаметром 30 и длиной $60 \mathrm{~mm}$, не имеющие рассеивающих свет включений. Выращенные кристаллы были однофазны и принадлежали к структурному типу тисонита, пр.гр. $P \overline{3} c 1$ (порошковый рентгеновский дифрактометр Rigaku MiniFlex 600, излучение $\mathrm{Cu} K_{\alpha}$ ). Параметры элементарной ячейки равны $a=7.1312(1), c=7.2877(1) \AA$ и хорошо согласуются с кристаллохимическими данными [12,13]. Измеренная методом гидростатического взвешивания плотность кристаллов $\rho=6.080(6) \mathrm{g} / \mathrm{cm}^{3}$ (теоретическая плотность $\rho_{X}=6.12 \mathrm{~g} / \mathrm{cm}^{3}$ ). 
Таблица 1. Параметры ионного переноса в кристалле $\mathrm{CeF}_{3}$ по разным кристаллографическим направлениям

\begin{tabular}{c|c|c}
\hline Направление & $\begin{array}{c}\text { Низкотемпературная } \\
\text { область }\end{array}$ & $\begin{array}{c}\text { Высокотемпературная } \\
\text { область }\end{array}$ \\
\hline \multirow{2}{*}{ ос1 $\overline{1} 0]$} & $A_{1}=9.8 \cdot 10^{3} \mathrm{SK} / \mathrm{cm}$ & $A_{2}=56 \mathrm{SK} / \mathrm{cm}$ \\
& $H_{\sigma, 1}=0.45 \mathrm{eV}$ & $H_{\sigma, 2}=0.26 \mathrm{eV}$ \\
& $\sigma_{\| a}=8.0 \cdot 10^{-7} \mathrm{~S} / \mathrm{cm}(300 \mathrm{~K})$ & $\sigma_{\| a}=5.7 \cdot 10^{-4} \mathrm{~S} / \mathrm{cm}(600 \mathrm{~K})$ \\
\hline \multirow{2}{*}{ ось $a$} & $A_{1}=1.5 \cdot 10^{4} \mathrm{SK} / \mathrm{cm}$ & $A_{2}=59 \mathrm{SK} / \mathrm{cm}$ \\
& $H_{\sigma, 1}=0.44 \mathrm{eV}$ & $H_{\sigma, 2}=0.22 \mathrm{eV}$ \\
& $\sigma_{\| c}=2.7 \cdot 10^{-6} \mathrm{~S} / \mathrm{cm}(300 \mathrm{~K})$ & $\sigma_{\| c}=1.2 \cdot 10^{-3} \mathrm{~S} / \mathrm{cm}(600 \mathrm{~K})$
\end{tabular}

Для кондуктометрических измерений из кристаллической були вырезались плоскопараллельные образцы толщиной $2 \mathrm{~mm}$, ориентированные по осям $a$ и $c$ эле-

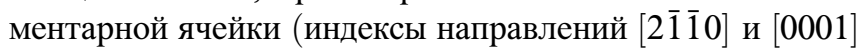
в тригональной четырехосной системе координат соответственно) с точностью не хуже 30'. Статическую электропроводность $\sigma_{d c}$ на постоянном токе определяли из спектров комплексного импеданса $Z^{*}(\omega)$ в диапазоне частот $1-3 \cdot 10^{7} \mathrm{~Hz}$ (установка Novocontrol-1200, прибор Alpha-A-impedance analyzer). В качестве контактов применяли платиновую пасту, площадь электродов составляла $33 \mathrm{~mm}^{2}$. Измерения $\sigma_{d c}$ проводили в инертной $(\mathrm{Ar})$ атмосфере в интервале температур 300-600 K с шагом $6 \mathrm{~K}$. Относительная погрешность измерений $\sigma_{d c}$ не превышала $2 \%$.

\section{3. Обсуждение результатов}

Температурные зависимости проводимости для образцов $\mathrm{CeF}_{3}$ по осям $a$ и $c$ показаны на рис. 1. Наиболее высокая электропроводность наблюдается вдоль оси $c$. Температурные зависимости $\sigma_{\| c}(T)$ и $\sigma_{\| a}(T)$ разбиваются на три участка: 300-355 K (низкие температуры), 355-545 K (средние температуры) и 545-600 К (высокие температуры). Низко- и высокотемпературные участки зависимостей $\sigma_{\| c}(T)$ и $\sigma_{\| a}(T)$ описываются уравнением Аррениуса-Френкеля

$$
\sigma_{d c} T=A \exp \left(-H_{\sigma} / k T\right),
$$

где $A-$ предэкспоненциальный множитель электропроводности, $H_{\sigma}$ - энтальпия активации процесса ионного переноса, $k$ - постоянная Больцмана и $T-$ температура. Параметры ионного переноса на этих участках приведены в табл. 1. На среднетемпературном участке происходит смена механизма ионного переноса, сопровождающаяся монотонным уменьшением энтальпии активации ионного транспорта с ростом температуры.

Полученные значения энтальпий активации ионного переноса $H_{\sigma, 1}$ и $H_{\sigma, 2}$ для кристалла $\mathrm{CeF}_{3}$ вдоль оси $c$ хорошо согласуются с электрофизическими данными $[8,14]$ и близки к активационным энтальпиям $H_{\sigma, 1}=0.34-0.46 \mathrm{eV}$ и $H_{\sigma, 2}=0.2-0.3 \mathrm{eV}$ [6-9] для изоструктурного $\mathrm{LaF}_{3}$. Можно видеть, что для тисонитовых кристаллов $\mathrm{CeF}_{3}$ (также как $\mathrm{LaF}_{3}$ ) выполняется

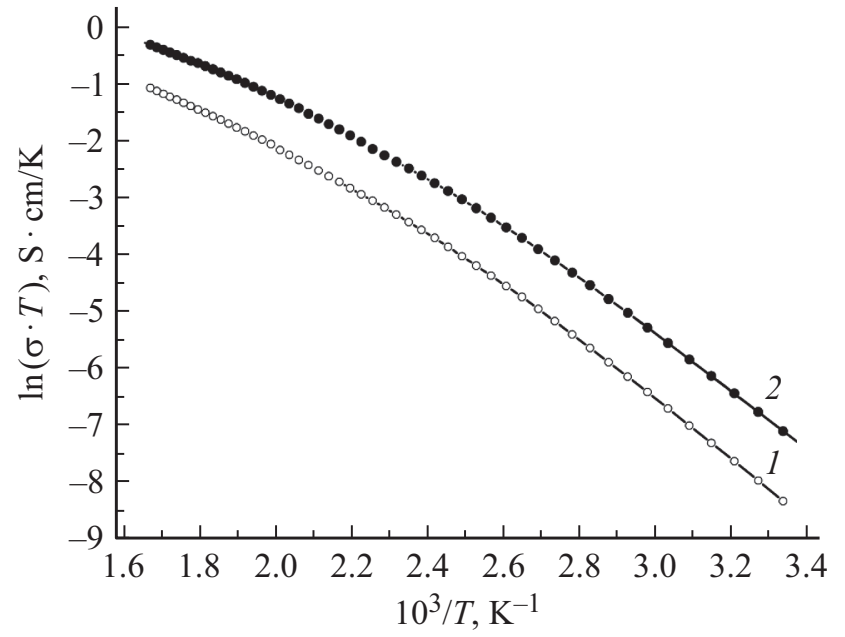

Рис. 1. Температурные зависимости ионной проводимости $\sigma_{d c}(T)$ по кристаллографическим осям кристалла $\mathrm{CeF}_{3}: 1-$ $\sigma_{\| a}, 2-\sigma_{\| c}$.

условие: $H_{\sigma, 1}>H_{\sigma, 2}$. Выполнение этого условия обусловлено тем, что в механизме ионного переноса для низко- и высокотемпературных областей электропроводности участвуют вакансии фтора, находящиеся в разных кристаллографических позициях тисонитовой структуры [9,15-18].

Отношения значений проводимостей, измеренных вдоль осей $c$ и $a$, убывают с температурой и составляют $\sigma_{\| c} / \sigma_{\| a}=3.4,2.4$ и 2.1 при 300, 500 и $600 \mathrm{~K}$ соответственно.

Экспериментальные данные по анизотропии $\sigma_{d c}$ для большого круга кристаллов $R \mathrm{~F}_{3}(R=\mathrm{La}, \mathrm{Ce})$ и твердых растворов $R_{1-y} M_{y} \mathrm{~F}_{3-y}(R=\mathrm{La}-\mathrm{Nd}, M=\mathrm{Sr}, \mathrm{Ba})$ сведены в табл. 2. Видно, что анизотропный эффект ионного переноса в индивидуальных трифторидах РЗЭ является слабым. Более того, в концентрированных тисонитовых твердых растворах $R_{1-y} M_{y} \mathrm{~F}_{3-y}(y>0.05)$ он подавляется $[19,20]$.

Ионная проводимость в тисонитовых трифторидах Р3Э обусловлена трансляционными перескоками ионов фтора в кристаллической решетке по вакансионному механизму [21]. Незначительная анизотропия $\sigma_{d c}$ в трифториде $\mathrm{CeF}_{3}$ указывает на то, что в его структуре нет 
Таблица 2. Анизотропия ионной проводимости во фторидных кристаллах со структурой тисонита

\begin{tabular}{|c|c|c|c|c|}
\hline Кристалл & $T, \mathrm{~K}$ & Анизотропия $\sigma_{d c}$ & $\sigma_{\| c}, \mathrm{~S} / \mathrm{cm}$ & Литература \\
\hline \multirow{4}{*}{$\mathrm{LaF}_{3}$} & \multirow{2}{*}{300} & $\sigma_{\| c} / \sigma_{\perp c}=1.6$ & $7 \cdot 10^{-7}$ & {$[7]$} \\
\hline & & $\sigma_{\| c} / \sigma_{\perp c}=2$ & $1 \cdot 10^{-6}$ & [9] \\
\hline & \multirow{2}{*}{500} & $\sigma_{\| c} / \sigma_{\perp c}=1.6$ & $1.5 \cdot 10^{-3}$ & {$[6]$} \\
\hline & & $\sigma_{\| c} / \sigma_{\perp c}=4$ & $3 \cdot 10^{-4}$ & {$[8]$} \\
\hline $\mathrm{La}_{0.896} \mathrm{Ba}_{0.104} \mathrm{~F}_{2.896}$ & 500 & $\sigma_{\| c} / \sigma_{\perp c}=1$ & $1 \cdot 10^{-2}$ & {$[18]$} \\
\hline $\mathrm{CeF}_{3}$ & $\begin{array}{l}300 \\
500 \\
\end{array}$ & $\begin{array}{l}\sigma_{\| c} / \sigma_{\| a}=3.4 \\
\sigma_{\| c} / \sigma_{\| a}=2.4\end{array}$ & $\begin{array}{l}2.7 \cdot 10^{-6} \\
5.6 \cdot 10^{-4} \\
\end{array}$ & $\begin{array}{c}\text { настоящая } \\
\text { работа }\end{array}$ \\
\hline $\mathrm{Ce}_{0.98} \mathrm{Sr}_{0.02} \mathrm{~F}_{2.98}$ & 320 & $\sigma_{\| c} / \sigma_{\perp c}=3-4$ & $1.7 \cdot 10^{-3}$ & \multirow{4}{*}[19]{} \\
\hline $\mathrm{Ce}_{0.92} \mathrm{Sr}_{0.08} \mathrm{~F}_{2.92}$ & 320 & $\sigma_{\| c} / \sigma_{\perp c}=1$ & $6 \cdot 10^{-4}$ & \\
\hline $\mathrm{Pr}_{0.98} \mathrm{Sr}_{0.02} \mathrm{~F}_{2.98}$ & 320 & $\sigma_{\| c} / \sigma_{\perp c}=3-4$ & $1.3 \cdot 10^{-3}$ & \\
\hline $\mathrm{Nd}_{0.85} \mathrm{Sr}_{0.15} \mathrm{~F}_{2.85}$ & 320 & $\sigma_{\| c} / \sigma_{\perp c}=1$ & $1.1 \cdot 10^{-7}$ & \\
\hline
\end{tabular}

выделенных каналов проводимости и он обладает квазитрехмерной $(3 D)$ электропроводностью. В бинарных системах $M \mathrm{~F}_{2}-R \mathrm{~F}_{3}(M=\mathrm{Ca}, \mathrm{Sr}, \mathrm{Ba} ; R=\mathrm{La}-\mathrm{Nd})$ существуют протяженные области гетеровалентных твердых растворов, что позволяет увеличить величину $\sigma_{d c}$ для $R \mathrm{~F}_{3}$ путем гетеровалентного замещения $[22,23]$.

Незначительная анизотропия $\sigma_{d c}$ обусловлена особенностями атомного строения трифторидов РЗЭ со структурой типа $\mathrm{LaF}_{3}$, которые являются диморфными [24]. Низкотемпературная форма относится к тригональной пр.гр. $P \overline{3} c 1(Z=6)$, высокотемпературная к гексагональной пр. гр. $P 6_{3} / m m c(Z=2)$. Тригональная структура имеет три анионные (фторные) подсистемы $\mathrm{F}_{1}: \mathrm{F}_{2}: \mathrm{F}_{3}=12: 4: 2$ (рис. 2). Путь наивысшей проводи- мости в тисонитовых трифторидах Р3Э имеет кристаллографическую направленность (ось $c$ ) [25]. В тригональном мотиве тисонита вдоль оси $c$ чередуются чисто анионные слои, образованные атомами $\mathrm{F}_{1}$, и катионанионные слои, содержащие близкие по динамическим свойствам атомы $\mathrm{F}_{2}$ и $\mathrm{F}_{3} \quad\left(\mathrm{~F}_{2} \approx \mathrm{F}_{3}\right)$ (рис. 2) [26]. При совпадении структурных позиций $\mathrm{F}_{2}=\mathrm{F}_{3}$ тригональная модификация тисонита переходит в гексагональную [27], для которой выполняется соотношение $\mathrm{F}_{1}: \mathrm{F}_{2,3}=2: 1$.

Анизотропия $\sigma_{d c}$ определяется неэквивалентностью позиций фтора $\left(\mathrm{F}_{1}, \mathrm{~F}_{2,3}\right)$ и частот прыжков ионов $\mathrm{F}^{-}$ в этих позициях $[8,28]$. Как показано [29] при низких температурах ионы $\mathrm{F}^{-}$в позициях $\mathrm{F}_{1}$ имеют более высокую частоту прыжков, чем в позициях $\mathrm{F}_{2,3}$. Перенос

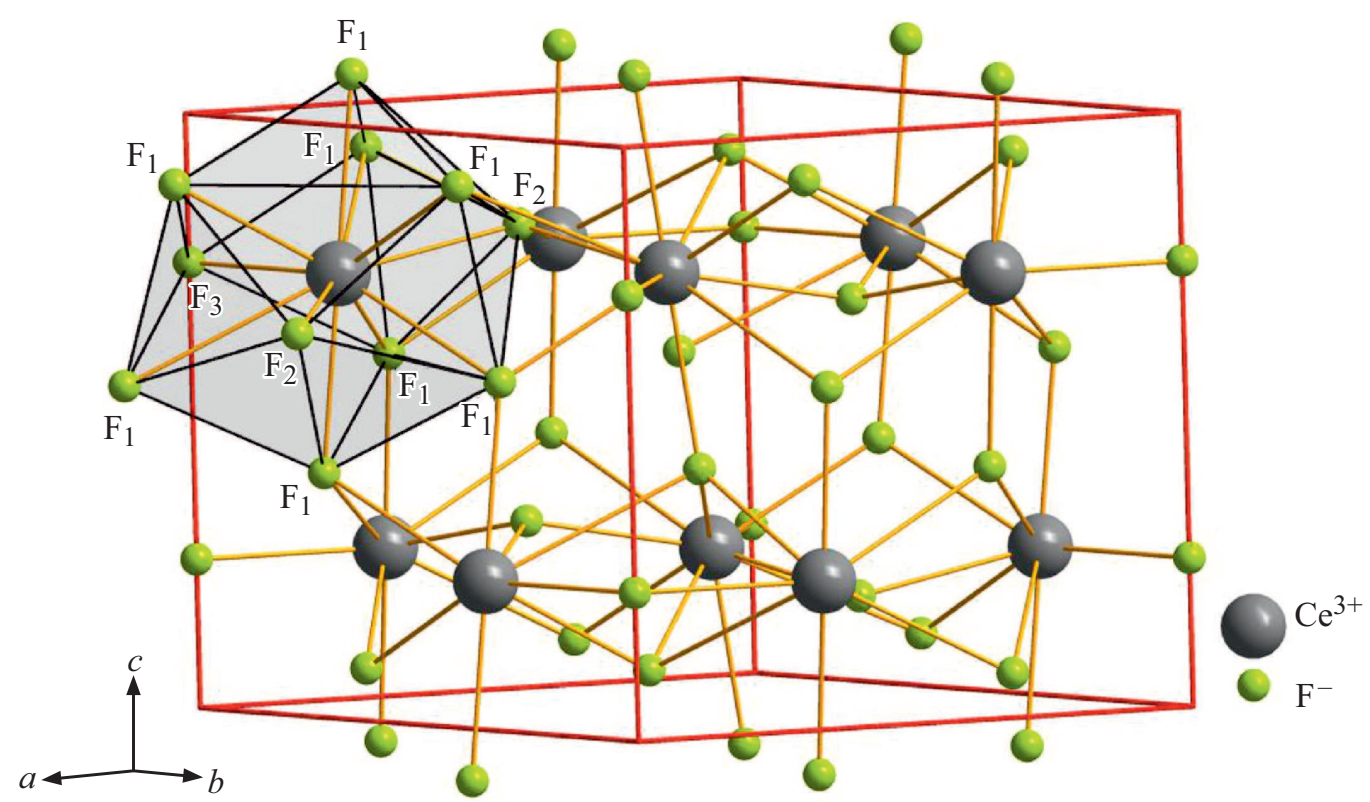

Pис. 2. Структура кристалла $\mathrm{CeF}_{3}$ : элементарная ячейка и $\mathrm{CeF}_{11}$ полиэдр. 
ионов фтора вдоль оси $c$ более быстрый, поскольку он осуществляется вакансиями фтора с участием позиций $\mathrm{F}_{1}$ и используются более короткие структурные участки для путей проводимости.

С ростом температуры анионный перенос происходит сначала во фторной подсистеме $\mathrm{F}_{1}[9,15,17]$, затем наблюдается обмен вакансиями фтора между подсистемами $\mathrm{F}_{1}$ и $\mathrm{F}_{2,3}\left(\mathrm{~F}_{2}+\mathrm{F}_{3}\right)$. Введение гетеровалентных катионов $M^{2+}$ стабилизирует гексагональную тисонитовую структуру в нестехиометрических фазах $R_{1-y} M_{y} \mathrm{~F}_{3-y}[30,31]$.

В индивидуальных трифторидах РЗЭ образуются термостимулированные дефекты по механизму Шоттки (катионные и фторные вакансии) [9,32]. Подвижными дефектами являются вакансии фтора, которые участвуют в фтор-ионном транспорте. Синтез анион-дефицитных твердых растворов $R_{1-y} M_{y} \mathrm{~F}_{3-y}$ способствует появлению „примесных“ вакансий фтора (носителей заряда) в структурах типа тисонита за счет гетеровалентных замещений катионов $M^{2+}$ на катионы РЗЭ $R^{3+}$. С кристаллохимической точки зрения $[33,34]$ высокая абсолютная величина $\sigma_{d c}$ трифторидов РЗЭ с тисонитовым типом структуры объясняется высоким координационным числом катионов $R^{3+}[35,36]$, которое равно 11. Высокая координация фторами катионов $\mathrm{Ce}^{3+}\left(\mathrm{CeF}_{11}\right.$ полиэдр, рис. 2) приводит к ослаблению химических связей „металл-фтор“ и увеличению подвижности ионов $\mathrm{F}^{-}$. Для сравнения в обладающих не столь высокой ионной электропроводностью трифторидах РЗЭ структурных типов $\beta-\mathrm{YF}_{3}$ и $\mathrm{ReO}_{3}$ [36] оно составляет 8-9 и 6 соответственно.

\section{4. Заключение}

Впервые проведены температурные измерения проводимости $\sigma_{d c}(T)$ кристаллов $\mathrm{CeF}_{3}$ по кристаллографическим осям $a$ и $c$ тригональной элементарной ячейки. В интервале $300-600 \mathrm{~K}$ наблюдается слабая анизотропия анионной проводимости: $\sigma_{\| c} / \sigma_{\| a}=2.1-3.4$. Трифториды РЗЭ структурного типа тисонита обладают $3 D$-электропроводностью из-за отсутствия каналов проводимости в тисонитовой структуре. Высокая ионная электропроводность по ионам фтора у фторидных материалов на основе $R \mathrm{~F}_{3}$ реализуется из-за высокой координации фторами катионов, образующих структурный каркас, и при условии наличия значительной концентрации анионных вакансий вследствие гетеровалентных замещений.

\section{Финансирование работы}

Работа выполнена при поддержке Министерства науки и высшего образования в рамках выполнения работ по Государственному заданию Федерального научноисследовательского центра „Кристаллография и фотоника“ РАН с использованием оборудования ЦКП
ФНИЦ „Кристаллография и фотоника“ РАН (проект RFMEFI62119X0035).

\section{Конфликт интересов}

Авторы заявляют, что у них нет конфликта интересов.

\section{Список литературы}

[1] G. Karkera, M. Anji Reddy, M. Fichtner. J. Power Sources 481, 228877 (2021).

[2] B.P. Sobolev, N.I. Sorokin, N.B. Bolotina. Photonic \& electronic properties of fluoride materials / Eds A. Tressaud, K. Poeppelmeier. Elsevier, Amsterdam (2016) P. 465.

[3] J. Chable, B. Dieudonne, M. Body, C. Legein, M.P. CrosnierMopez, C. Galven, F. Mauvy, E. Durand, S. Fourcade, D. Sheptyakov, M. Leblanc, V. Maisonneuve, A. Demourgues. Dalton Trans. (2017). DOI: 10.1039/c6dt04714a.

[4] M. Kabayashi, M. Ishii, E.A. Krivandina, M.M. Litvinov, A.I. Peresypkin, Yu.D. Prokoshkin, V.I. Rykalin, B.P. Sobolev, K. Takamatsu, V.G. Vasil'chenko. Nucl. Instrum. Meth. A. 302, 443 (1991).

[5] Б.П. Соболев, Т.М. Глушкова, Е.А. Кривандина, З.И. Жмурова. Кристаллография 57, 914 (2012).

[6] A.V. Chadwick, D.S. Hope, G. Jaroszkiewicz, J.H. Strange. Fast Ion Transport in Solids. Eds. P. Vashishta, N. Mundy, G.K. Shenoy. North-Holland, Amsterdam (1979) P. 683.

[7] J. Schoonman, G. Oversluizen, K.E.D. Wapenaar. Solid State Ionics 1, 211 (1980).

[8] C. Hoff, H.D. Wiemhofer, O. Glumov, I.V. Murin. Solid State Ionics 101-103, 445 (1997).

[9] V.V. Sinitsyn, O. Lips, A.F. Privalov, F. Fujara, I.V. Murin. J. Phys. Chem. Solids 64, 1201 (2003).

[10] Н.Л. Сизова, Д.Н. Каримов, Т.Б. Косова, Д.С. Лисовенко. Кристаллография 64, 935 (2009).

[11] R. Laiho, M. Lakkisto. Phyl. Mag. B 48, 203 (1983).

[12] O. Greis, T. Petzel. Z. Anorgan. Allgem. Chem. 403, 1 (1974).

[13] Н.Б. Болотина, Т.С. Черная, И.А. Верин, О.Н. Хрыкина, Б.П. Соболев. Кристаллография 61, 36 (2016).

[14] А.Л. Пирозерский, Е.В. Чарная, Е.Л. Лебедева, Е.Н. Латышева, Н.И. Сорокин, В.Ф. Криворотов, А.А. Фридман. Электрохимия 47, 332 (2011)

[15] C. Tien, E.V. Charnaya, A.B. Sherman. ФTT 46, 1578 (2004).

[16] A.F. Aaders, A. Polman, A.F.M. Arts, H.W. de Wijn. Solid State Ionics 9-10, 539 (1983).

[17] М.Г. Изосимова, А.И. Лившиц, В.М. Бузник, Ю.В. Амелин, И.В. Мурин, А.Н. Мурин. Изв. АН СССР. Неорган. материалы 23, 2056 (1987).

[18] F.C. Case, P.P. Mahendroo. J. Phys. Chem. Solids 42, 385 (1981).

[19] A. Roos, F.C.M. van de Pol, R. Keim, J. Schoonman. Solid State Ionics 13, 191 (1984).

[20] Н.И. Сорокин, Б.П. Соболев. Электрохимия 43, 420 (2007).

[21] А.Ф. Привалов, И.В. Мурин. ФТТ 41, 1616 (1999).

[22] Б.П. Соболев, Н.И. Сорокин, Е.А. Кривандина, З.И. Жмурова. Кристаллография 59, 609 (2014).

[23] Н.И. Сорокин, Б.П. Соболев, Е.А. Кривандина, З.И. Жмурова. Кристаллография 60, 123 (2015).

[24] O. Greis, M.S.R. Cader. Thermochim. Acta 87, 145 (1985).

[25] I. Branch, H. Schulz. Solid State Ionics 15, 135 (1985) 
[26] M. Mansmann. Z. Kristallogr. 122, 375 (1965).

[27] K. Schlyter. Arkiv Kemi. 5, 73 (1953).

[28] D.R. Franceschetti, P.C. Shipe. Solid State Ionics 11, 285 (1984)

[29] A.F. Privalov, H.-M. Vith, I.V. Murin. J. Phys. Condens. Matter. 6, 8237 (1984)

[30] Л.П. Отрощенко, В.Б. Александров, Б.А. Максимов, В.И. Симонов, Б.П. Соболев. Кристаллография 30, 658 (1985).

[31] Н.Б. Болотина, Т.С. Черная, А.И. Калюканов, И.А. Верин, Н.И. Сорокин, Л.Е. Фыкин, Н.Н. Исакова, Б.П. Соболев. Кристаллография 60, 391 (2015).

[32] A. Sher, R. Solomon, K. Lee, M.W. Muller. Phys. Rev. 144, $593(1966)$

[33] Н.И. Сорокин, Д.Н. Каримов, В.В. Гребенев, Б.П. Соболев. Кристаллография 61, 270 (2016).

[34] J.M. Reau, J. Grannec. Inorg. Solid Fluorides / Ed. P. Hagenmuller. Academic Press, N.Y. (1985) P. 423.

[35] A.K. Cheetham, B.E.F. Fender, H. Fuess, A.F. Wright. Acta Cryst. B. 32, 94 (1976).

[36] Л.С. Гарашина, Б.П. Соболев, В.Б. Александров, Ю.С. Вишняков. Кристаллография 25, 294 (1980).

Редактор К.В. Емцев 